\title{
Priority-Based Node Selection and Scheduling for Wireless Multimedia Sensor Networks
}

\author{
Mohammad Alaei ${ }^{1,2}$, Jose Maria Barcelo-Ordinas ${ }^{1}$ \\ ${ }^{1}$ Computer Architecture Department \\ ${ }^{2}$ Computer Engineering Department \\ Technical University of Catalonia (UPC) \\ Shahid Bahonar University of Kerman \\ Barcelona, Spain \\ $\{$ malaei , joseb $\} @$ ac.upc.edu \\ Kerman, Iran \\ m_alaei@mail.uk.ac.ir
}

\begin{abstract}
A critical aspect of applications with wireless sensor networks is network lifetime. Sensing and communications consume energy particularly in wireless multimedia sensor networks (WMSN) due to huge amount of data generated by the multimedia sensors. Therefore, judicious power management and sensor scheduling can effectively extend network lifetime. In this paper we consider the problem of scheduling multimedia sensor activities to maximize network lifetime. The environment is divided in domains monitored by clusters of multimedia sensor nodes. Network lifetime increment is achieved by cooperation between multimedia sensors in two priority-based ways: Intracluster cooperation and Inter-cluster cooperation. We will see that the lifetime of cluster nodes is considerably increased under the proposed node selection and scheduling procedures. As for big clusters, the lifetime even is prolonged to 5.5 times with respect to the ordinary un-cooperative node awakening.
\end{abstract}

Keywords - Wireless Multimedia Sensor Network; Energy Efficiency; Sensor Scheduling; Cooperation; Clustered Network.

\section{INTRODUCTION}

Wireless Multimedia Sensor Networks (WMSNs) [1] are networks of resource-constrained wireless devices that can retrieve multimedia content such as still images or video and audio streams from the environment. WMSNs will also be able to store, process in real-time, correlate, and fuse multimedia data originated from heterogeneous sources. Multimedia nodes are constrained in terms of battery, bandwidth, memory, processing capability, and achievable data rate.

On the other hand, the volume of multimedia data, particularly images sensed by the camera sensors, is much larger than scalar sensors [1][2]. In-node processing avoids transmitting all data gathered by the multimedia nodes towards the sink or the base station and thus reduces the traffic and resource usage of the network. The constraint of power and processing ability also limits nodes to accomplish complicated processing on the huge amount of sensed multimedia data [3]. Applying cooperation among nodes or groups of nodes for multimedia sensing and processing is an effective manner to manage the power consumption and thus the life-time of the network.

Recently, the availability of low-cost hardware and developments in low power CMOS digital cameras are enabling the development of embedded multimedia nodes.
Having dense deployments of low cost, low power and low resolution camera sensors in WMSNs to sense and monitor the environment especially in some applications that employ a random deployed network such as battlefield surveillance, environment monitoring, biological detection and agricultural fields. But, due to the specific properties of wireless multimedia sensor networks in both aspects of special directional sensing region of multimedia nodes, Field of View (FoV), and huge amount of multimedia data generated by the nodes which have to be transmitted or processed, there are several challenges in these networks [4]. Here, we emphasize on energy efficiency and cooperative coverage.

Papers [5] and [6] show how multimedia nodes of a randomly deployed WMSN are categorized in clusters considering the FoV as the criterion of clustering. If the FoVs of two nodes have a wide common area, sensors are grouped in a cluster since they obtain a similar vision of the monitored area. The established clusters in [5] are disjoint and nonoverlapping while in [6] they overlap each other with common nodes.

The contribution of the present work is planning schedules for node awakening in WMSNs in order to enable cooperative multimedia monitoring and to maximize network lifetime. We first consider a network consisting of disjoint clusters. Nodes belonging to the same cluster cooperate in multimedia tasks and work in a priority-based way. The node having the most energy level of a given cluster acts in the current monitoring period while other members of the cluster are saving their energy in the sleep mode and waiting for their round to play their role. We second consider a network with overlapping clusters. In addition to the intra-cluster cooperation, intercluster coordination can increase the level of cooperation and thus of network lifetime, by selecting nodes that are common to more than one cluster to monitor the area. It is to say, for each monitoring period, the minimum number of nodes covering all established clusters are selected and awakened to monitor the area. The priority of a node for monitoring is determined considering the four following parameters: (i) The residual energy of the node, (ii) The membership-degree of the node (i.e, the number of clusters that the node belongs to), (iii) Working mode of the node in the previous monitoring period (i.e., activating a node in two consecutive periods is not desirable because of the repetitious FoVs), (iv) Existence of 
other selected nodes for activating in the current monitoring period from the same cluster that the node belongs to (i.e., selecting more than one node from a cluster for a period is not desirable). We will show how the proposed node selection procedure for intra and inter-cluster cooperative monitoring develops the energy conservation capability of nodes and thus prolongs the network lifetime.

The remainder of this paper is organized as follows. Section II discusses the related work. Section III presents the two proposed node selection and scheduling for cooperative monitoring schemes, first with intra-cluster cooperation and the second offering intra and inter-cluster cooperation. Section IV proceeds to the comparisons and evaluation of the proposed algorithms and finally conclusions are derived in section V.

\section{RELATED WORK}

Lifetime prolongation has been studied in great depth in the field of wireless sensor networks (WSN). An important method for prolonging the network lifetime for the area coverage problem is to determine a localized and distributed protocol for selecting the set of active sensor nodes. Several works have proceeded to this research area for scalar sensor networks in which a sensor covers a target if the Euclidean distance between the sensor and target is smaller or equal with a predefined sensing range. The authors of [7] introduce a model of the target coverage problem for maximizing the network lifetime by organizing the scalar sensor nodes in set covers. Each node sensing the area being a disk centred at the sensor. They consider a number of targets with known locations that need to be continuously covered and a large number of sensors randomly deployed closed to the targets. Two solutions have been proposed for the Maximum Set Covering problem using linear programming and a greedy heuristic algorithm. But, the sensing region of multimedia nodes is very different from scalar nodes in WSNs [4]. Each multimedia node has a FoV and only can capture images from the objects within that region. Video cameras capture images of objects of a region that are not necessarily in the camera's vicinity. The object covered by the camera can be distant from the camera and the captured images will depend on the relative positions and orientations of the cameras towards the observed object.

In [8], the authors propose a distributed solution to camera actuation problem in WMSNs. The idea is for each camera sensor to utilize the number of scalar sensors which detected an event within its FoV and exchange this information with the neighbouring camera sensors to determine the possible coverage overlaps. Counting the number of scalar sensors is the way to determine the size of the event areas. Based on such information, the camera sensors which hear from a higher number of scalar sensors will be given priority in actuation.

The joint effect of multiple correlated cameras in WMSNs is studied in [9]. The authors propose an entropy-based analytical framework to measure the amount of information provided by multiple cameras in WMSNs. A correlation-based camera selection scheme is also introduced to select cameras from sensor networks under distortion constraints.

There are some researches on target surveillance application of WMSNs. A distributed network structure is defined in [10] with honeycomb configuration, where the coverage efficiency is justified with the limited communication range. With the historical target positions, the target position is forecasted by ARMA-RBFN (Auto Regressive Moving Average-Radial Basis Function Network), where RBFN is utilized to compensate the ARMA forecasting. The work [11] proposes a dynamic cluster based node collaboration scheme to deal with the mobile target tracking problem in wireless camera sensor networks. The authors first develop a nonlinear localization-oriented sensing model for camera sensors by taking the observation noisy into account. Based on this sensing model, the Sequential Monte Carlo (SMC) technique is applied for electing cluster head and selecting cluster members during the tracking process. In [12], the maximal breach is computed for a target which corresponds to worstcase camera coverage. An optimal algorithm is presented for such purpose by considering a more generalized model for representing the FoV.

Hierarchical network architectures have been presented with multiple tiers having assigned tasks for each tier as in SensEye [13], object detection in first tier, can be achieved with lightweight low-cost cameras. Cyclops [14] is an example of appropriate sensors in this tier. The sensor nodes are dutycycled and woken up periodically to capture an image and detect presence of new objects in the area independent of each other and thus there is no cooperation among nodes. Object detection procedure is performed via simple frame differencing.

Finally, in this paper we will define node selection mechanisms for duty-cycling cooperative multimedia monitoring using FoV-overlapping based clustering algorithms. FoV overlapping and disjoint clusters formation (Single-Cluster Membership, SCM) for WMSN is proposed in [5]. FoV overlapping and overlapping-clusters formation (Multi-Cluster Membership, MCM) for WMSN is proposed in [6]. Although, we will use these clustering schemes, the purpose of this paper is to propose procedures for cooperative environment monitoring with intra-cluster and also intra \& inter-cluster cooperation for disjoint and overlapping clusters without being specially tied to the SCM and MCM cluster formation mechanisms.

\section{NODE SELECTION AND SCHEDULING PROCEDURES FOR COOPERATIVE MONITORING IN CLUSTERED WMSNS}

Today, because of developments in low cost, low power, low resolution camera sensors such as CMOS cameras, dense random deployments of them are applicable. This kind of deployment is more efficient than sparse ones of high power, high cost, high resolution cameras since with crashing a node or facing with an obstacle (which is highly possible in random deployments) the network will not be seriously damaged. However, in dense random deployed WMSNs many of the nodes have overlapping FoVs, yielding redundant multimedia sensing and/or processing that has as a consequence the wasting of power in the network.

Clustering the multimedia nodes with overlapping FoVs and scheduling the cluster members having highly overlapped FoVs, is a way to avoid redundant sensing of the environment and thus saving energy. On the other hand, FoV-based clustering also allows multi-view monitoring by overlapping 
cluster members monitoring the same area/object concurrently. In this way, each member takes the view from its perspective at the cost of all cluster members spend simultaneously sensing, processing and transmission energy. In this case, power saving will not be achieved due to the multiple images taken by the overlapping nodes. In any case, the scheduling of nodes in the clusters based on overlapping FoV is a challenge and a key objective in the design of monitoring applications.

In this section, we propose two cooperative procedures for node selection and scheduling to monitor the environment by a WMSN. In the first mechanism, cooperation and thus scheduling is defined only for members of the same cluster and is thought for those clustering algorithms that cluster nodes in such a way that a node can only belong to one cluster. That means that all the clusters are disjoint clusters. An example of this kind of disjoint clustering mechanisms can be found in [5] and [15]. In the second mechanism, cooperation is defined for nodes that belong to the same cluster and for nodes that belong to more than one cluster. That means that there will be clusters that are disjoint but also there can be clusters that have common nodes and overlap each other. An example of this kind of clustering mechanisms can be found in [6].

To be distributed and localized are important properties of a node scheduling mechanism, as they better adapt to a scalable and dynamic network topology. Both of the proposed monitoring schemes are distributed and localized in clusters. Finally, monitoring is organized in duty-cycled periods, and the set of active sensor nodes is decided at the beginning of each period.

In this paper we are concerned with designing the node selection and scheduling mechanism, and do not address the problem of selecting which protocol is used for data gathering. Without loss of generality, to efficiently transmit data from the detector sensors to the sink, a mechanism like PEGASIS [16] can be used.

\section{A. Intra-Cluster Cooperative Scheme (ICC)}

Single-Cluster Membership (SCM) is a clustering method for multimedia sensor nodes, [5], that group nodes in disjoint and non-overlapping clusters based on their overlapping FoVs. If the FoVs of two nodes intersect each other in a wide area, they join to the same cluster. Therefore, nodes belonging to the same cluster sense the environment with a high overlapping level. In order to define a cooperative monitoring scheme, we employ the cluster members to monitor the environment sequentially. The sensing environment is divided in domains among clusters and each cluster periodically monitors its domain in a duty-cycled manner. Fig. 1 shows an example of 5 clusters established on 11 nodes.

Let us define a monitoring period $(\mathrm{T})$ in which a cluster member that is activated by its cluster-head, monitors its area by capturing an image and surveying the presence of a new object/event in the captured image by performing an object detection procedure. We assume that all nodes of the network have the same initial stored energy at the time of deployment.

Accordingly, the monitoring procedure based on this node clustering will be as follows: at the beginning of each monitoring period $(\mathrm{T})$ and at each cluster, the member having

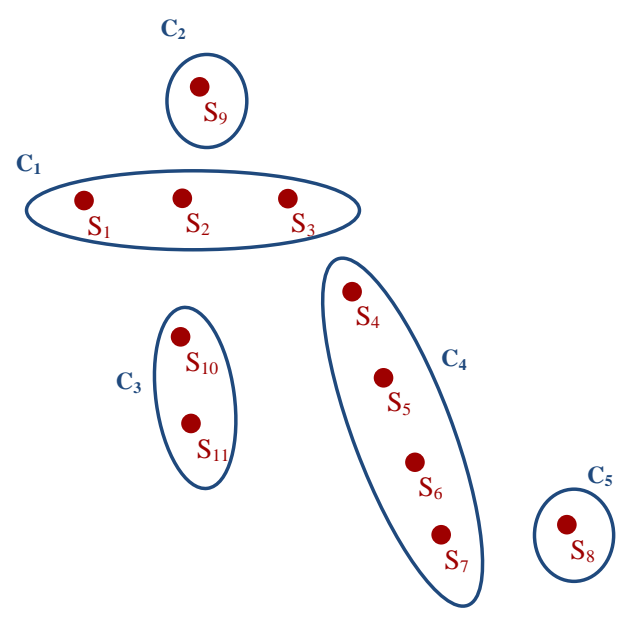

Figure 1. An example of disjoint clusters established on 11 nodes, single nodes that do not have enough overlap with other nodes, make clusters with only one member.

the maximum residual energy level is selected by its clusterhead as the representative of the cluster for sensing the environment while the other members of the cluster are kept in the sleep mode. The awakened member captures an image from its FoV and surveys the presence of the object/event and go back to the sleep mode. Each cluster-head records the current energy level of all members of its cluster in a register and refreshes that in each monitoring period with the new values according to the sleeping or active states of the cluster members in the current period. During each monitoring period, the awakened and sleeper nodes spend their energy proportional to their working state.

Fig. 2 shows the energies consumed in the monitoring $\left(E_{M}\right)$ and sleeping $\left(E_{S}\right)$ modes by a node. Awakened members that monitor the area (capturing and detection) in the current period, consume their energy for monitoring while other nodes of the network that remain in the sleep mode during the $\mathrm{T}$, just use the power of sleeping state. Thus, at the end of each period, the total energy consumed in all nodes of the network is reduced with respect a non-cooperative scheme in which each node awakes and monitors the area [13].

Since in every period each cluster-head determines a member to monitor the area, the number of assigned nodes to be awakened is the same as the number of clusters in the network. The scale of cooperation in each cluster depends on the cluster size; in a cluster with more number of nodes, each node can save more amount of energy during the times it sleeps while the other cluster members are activated and monitor the area. The single nodes in the network that do not have enough overlap with others to join to the established clusters are programmed to awake every $\mathrm{T}$ to monitor their area and will be the first ones to die.

When a sensor node detects a new object/event, it notifies the cluster-head and also sends the image toward the sink. Therefore, some amount of energy is consumed in nodes for packet sending and forwarding of detected data and thus the residual energy of nodes is affected by that, and thus the cluster-heads need to update the residual energy level values $\left(E_{R}\right)$ of the members in their register to have a real view of their energy for node selection. On the other hand, as the node 
selection is accomplished in clusters in a distributed manner, for synchronizing the members and also for cluster maintenance, the cluster-heads make use of a message exchanging scheme: cluster nodes receive periodical messages from the cluster-head and acknowledge them not only with timing information but also with their current energy level. The cluster-heads in addition to synchronize the members, refresh their register of members energy information, detect new members or detect members that have died. This periodical message exchanging belongs to the radio subsystem and it is independent of the sensing subsystem thus is independent of waking up the sensor to monitor the area. Thus, the cluster-head may resynchronize cluster members without need of waking up the sensing subsystem. In case the cluster-head is depleting its energy, the highest energy level member of the cluster will be the new cluster-head. We use the proposed scheme in [17] for synchronization and cluster maintenance.

Since the clusters are disjoint, the monitoring scheme only offers intra-cluster cooperation. Fig. 3 shows an example corresponding to Fig.1 on how the selected cluster representatives are awakened in each monitoring period under the intra-cluster cooperative algorithm. Nodes $S_{8}$ and $S_{9}$ are awakened every $\mathrm{T}$ since they belong to cluster with only one member while in other clusters consisting of several nodes, the members cooperatively monitor the area. For instance, in the cluster $\mathrm{C}_{4}$ consisting of nodes $\mathrm{S}_{4}, \mathrm{~S}_{5}, \mathrm{~S}_{6}$ and $\mathrm{S}_{7}$, each node will be awakened every $4 \cdot \mathrm{T}$, being this cluster the one with the most energy saving potential among the clusters of the example. Finally, in Table 1, the Intra-cluster cooperation algorithm is described.

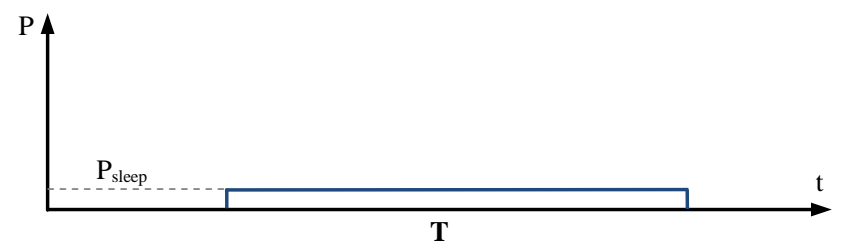

(a)

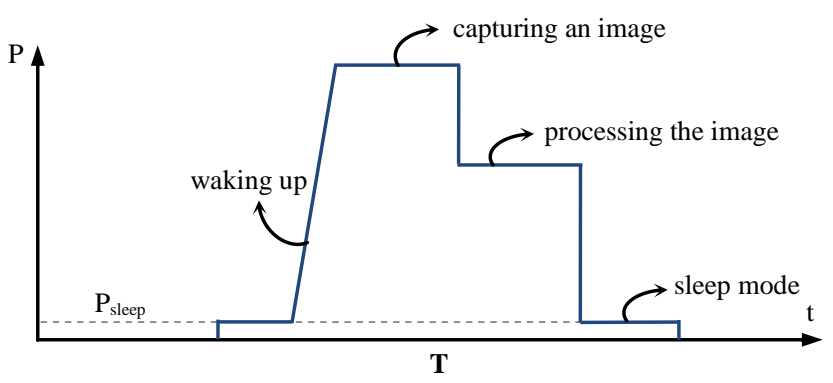

(b)

Figure 2.(a). The energy consumed by a sleeper node during the period $\mathrm{T}\left(\mathrm{E}_{\mathrm{S}}\right)$, the node consume $\mathrm{P}_{\text {sleep }}$ during the period, (b). The energy consumed by an activated node during the period $\mathrm{T}\left(\mathrm{E}_{\mathrm{M}}\right)$, consisting of the energy used for waking up, capturing image, processing the captured image (in our case, Object Detection) and remaining in sleep mode until the next period.

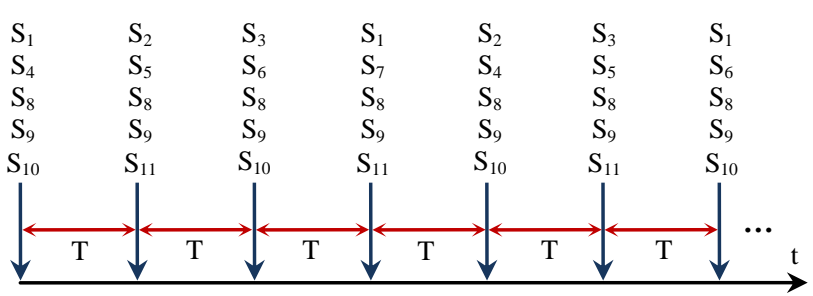

Figure 3. The selected nodes by Intra-Cluster cooperation (ICC) to monitor the area

Table 1. The first Scheduling Algorithm

\begin{tabular}{cc}
\hline & Intra-Cluster Cooperation (ICC) \\
\hline 1: & For all clusters: // All clusters in parallel // \\
2: & Active-node $\leftarrow$ node with the maximum $\mathrm{E}_{\mathrm{R}} / / E_{R}$ is the \\
& remainder energy in the node // \\
3: & Cluster-head awaken Active-node \\
4: & E $_{\mathrm{R}}$ Active-node $=\mathrm{E}_{\mathrm{R}}$ Active-node $-\mathrm{E}_{\mathrm{M}} / / E_{M}$ is the energy consumed \\
& by an active node during $T / /$ \\
5: & For all sleeper nodes \\
6: & $\quad \mathrm{E}_{\mathrm{R}}=\mathrm{E}_{\mathrm{R}}-\mathrm{E}_{\mathrm{S}} / / E_{S}$ is the energy consumed by a sleeper \\
7: & Endeduring the $T / /$ \\
8: & Delay $(\mathrm{T})$ \\
9: & Goto 2 \\
10: & End-For \\
\hline
\end{tabular}

\section{B. Intra and Inter-Cluster Cooperative Scheme (IICC)}

Multi-Cluster-Membership (MCM) is a clustering method for multimedia sensor nodes, [6], that establishes overlapping clusters consisting of multimedia nodes. In this clustering method, a node may belong to more than one cluster. In other words, clusters intersect each other by common nodes. It is clear that in the ICC scheme (section III.A) one node per cluster is awakened per monitoring period while in this scheme there can be a number of awakened sensors less or equal than the number of clusters. Thus, here comes the power savings.

In order to plan a cooperative monitoring scheme, we have to take into account that selecting nodes that only belong to one cluster will yield in intra-cluster monitoring. However, selecting a node that belongs to more than one cluster means that the common node monitors simultaneously the area corresponding to the clusters the node belongs to. We call this cooperation as inter-cluster cooperation.

Introducing inter-cluster cooperation is challenging as it can be observed in Fig. 4 in which several nodes act as common nodes. Let us consider node $\mathrm{S}_{2}$ that belongs to cluster $\mathrm{C}_{1}, \mathrm{C}_{2}$ and $\mathrm{C}_{3}$. The cardinality (i.e., number of nodes) of each cluster is $\left|\mathrm{C}_{1}\right|=4,\left|\mathrm{C}_{2}\right|=2,\left|\mathrm{C}_{3}\right|=3$. Selecting nodes sequentially as it was done in section 3.A would imply that node $S_{2}$ would be awakened more times than the other nodes, Fig. 5. For example, using the notation $C_{x}=\left\{S_{y}\right\}$ (i.e, cluster $C_{x}$ awakes node $\mathrm{S}_{\mathrm{y}}$ ), in the first $\mathrm{T}: \mathrm{C}_{1}=\left\{\mathrm{S}_{1}\right\}, \mathrm{C}_{2}=\left\{\mathrm{S}_{2}\right\}, \mathrm{C}_{3}=\left\{\mathrm{S}_{2}\right\}$, in the second period $\mathrm{T}: \mathrm{C}_{1}=\left\{\mathrm{S}_{2}\right\}, \mathrm{C}_{2}=\left\{\mathrm{S}_{9}\right\}, \mathrm{C}_{3}=\left\{\mathrm{S}_{10}\right\}$ and in the third period $\mathrm{T}: \mathrm{C}_{1}=\left\{\mathrm{S}_{3}\right\}, \mathrm{C}_{2}=\left\{\mathrm{S}_{2}\right\}, \mathrm{C}_{3}=\left\{\mathrm{S}_{11}\right\}$, etc. As it can be observed, the clusters have no knowledge of which nodes are awaked by other clusters. That means that common nodes are selected in a non-optimal way, more times than necessary 
times, and thus will die earlier than if an intelligent intercluster scheduling mechanism is chosen. In other words, common nodes are nodes with higher membership degree $\left(\mathrm{MEM}_{\text {DEGREE }}\right)$ than the other nodes. The membership degree is defined as the number of clusters a node belongs to and in the case of the example of Fig. 4, $\operatorname{MEM}_{\text {DEGREE }}\left(S_{2}\right)=3$.

The key point of an intelligent scheduling procedure for this kind of cluster memberships is that one in which common nodes are selected more times than others since they cover areas for several clusters but not so many times to deplete their batteries faster than other members of the clusters this nodes belongs to. Thus, we aim to defining a scheduling scheme that monitors the environment in a cooperative manner satisfying the following conditions:

(i) Awakening the minimum number of nodes in each period of monitoring to cover all clusters.

(ii) Prolonging the network lifetime and balancing the energy level of nodes.

(iii) Avoiding repeated FoVs in consecutive monitoring periods.

In order to schedule cluster members, we define two levels of priority for each multimedia node. The sum of the two priority values of each node determines its priority to monitor the environment for each monitoring period. The first level of priority, $\mathrm{P}^{\mathrm{L}_{1}}$, is a static value during each period and aims to choose the nodes with highest residual energy and membership-degree for monitoring the environment during the current monitoring period. That is determined from the residual energy in the node and also the membership-degree of the node. A node with a higher membership-degree is a member of a larger number of clusters than other nodes, thus it can monitor the area on behalf of more number of clusters and thus yields power savings in more number of members of its mother clusters. Therefore, selecting a high membershipdegree node always is advantageous since it covers a large number of clusters. However, the remainder energy of a node is quite important to select it for monitoring. Each awaken node spends its energy for monitoring the area, thus in addition to covering all clusters with the minimum number of awakened nodes, awakening nodes having higher level of residual energy is a criterion.

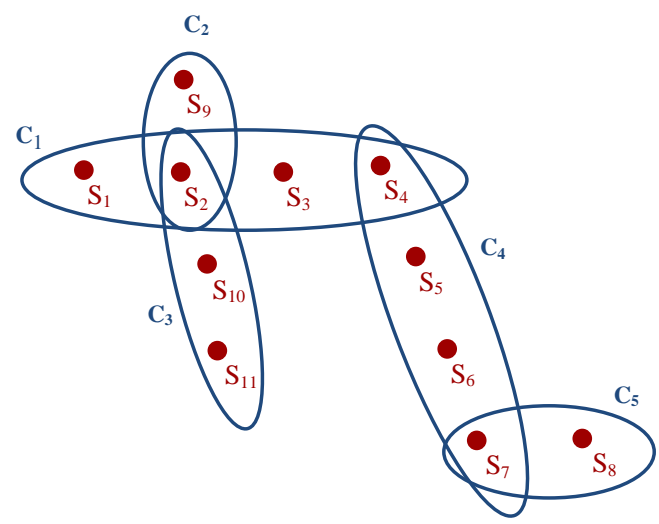

Figure 4. Overlapping clusters, $\mathrm{S}_{2}, \mathrm{~S}_{4}, \mathrm{~S}_{7}$ : Common nodes as the linkages for inter-cluster coordinating

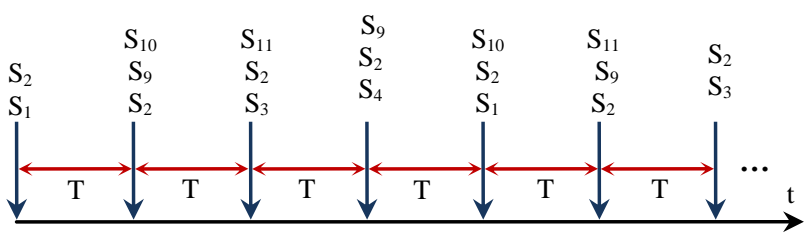

Figure 5. An example of timing for monitoring the area of clusters $C_{1}, C_{2}, C_{3}$ using a sequential mechanism (only nodes of clusters $C_{1}, C_{2}$ and $C_{3}$ are shown.)

$\mathrm{P}_{\mathrm{i}}^{\mathrm{L}_{1}}=\mathrm{E}_{\mathrm{R}_{\mathrm{i}}} / \mathrm{E}_{\mathrm{M}}+\left(\mathrm{MEM}_{\text {DEGREE }}-1\right), \forall \mathrm{S}_{\mathrm{i}}$

where $E_{R}$ is the residual energy, $E_{M}$ is the energy consumed by an active node during period $T$ (Fig. 2) and $E_{R} / E_{M}$ is the number of times that the node can monitor the area from its perspective considering its residual energy.

The second level of priority, $\mathrm{P}^{\mathrm{L}_{2}}$, is chosen to avoid selecting the same FoVs in consecutive periods and also to avoid selecting nodes from the covered clusters in the current T. $\mathrm{P}^{\mathrm{L}_{2}}$ is preset at the end of each period and during each period may be decreased according to the following rules:

(i) If a node was selected in the previous period for monitoring the environment, in the current period the node has a negative unit in its second level of priority. The aim followed of adding this negative unit is to avoid sensing the same FoVs in consecutive periods of monitoring when we can find other members having high priority to be selected for monitoring the area.

Presetting $\mathrm{P}^{\mathrm{L}_{2}}$ at the end of each period for the next period:

$\mathrm{P}_{\mathrm{i}}^{\mathrm{L}_{2}}=0, \forall \mathrm{S}_{\mathrm{i}}$

$\mathrm{P}_{\mathrm{i}}^{\mathrm{L}_{2}}=\mathrm{P}_{\mathrm{i}}^{\mathrm{L}_{2}}-1$, if $\mathrm{S}_{\mathrm{i}} \in \mathrm{CSS}$

where CSS is the Covering Sensors Set, the set of sensors activated in the current monitoring period.

(ii) When a cluster is covered by one of its members, all members of the cluster get a negative unit of priority to avoid selecting members of covered clusters when there are qualified applicable nodes of un-covered clusters.

Decreasing the $\mathrm{P}^{\mathrm{L}_{2}}$ during a period:

$\mathrm{P}_{\mathrm{i}}^{\mathrm{L}_{2}}=\mathrm{P}_{\mathrm{i}}^{\mathrm{L}_{2}}-1, \forall \mathrm{S}_{\mathrm{i}} \in$ the covered cluster

whenever a selected sensor $\left(S_{j}\right)$ covers the cluster that includes $S_{i}$

$\mathrm{P}_{\mathrm{i}}=\mathrm{P}_{\mathrm{i}}^{\mathrm{L}_{1}}+\mathrm{P}_{\mathrm{i}}^{\mathrm{L}_{2}}$

We assume the same initial energy level for all nodes. At the beginning of each period, the cluster-heads select and assign the members of Covering Sensors Set (CSS) of multimedia nodes having highest priorities to cover all clusters. Each cluster has a representative in the CSS while a selected node may represent more than one cluster according to its membership-degree. All the nodes of the CSS are awakened by their cluster-heads. In the case of a common node, it is awakened by the cluster-head of its smallest mothercluster. Awakened nodes monitor the area from their perspective and go to sleep mode again. During each period of 
monitoring, nodes of the CSS and the sleeper nodes, spend their energy proportional to their working state. Members of the CSS that monitor the area in the current period, consume their energy for monitoring $\left(\mathrm{E}_{\mathrm{M}}\right)$ while other nodes of the network that remain in the sleep mode during the $\mathrm{T}$, just use the power of sleeping state $\left(E_{S}\right)$, see Fig. 2. Thus, at the end of each period, the level of energy in all nodes of the network is reduced. Each cluster-head records the current energy and also the first and second levels of priority of all its cluster members in a register and refreshes that in each monitoring period with the new values. To refresh the register, the cluster-heads recompute the energy level and also the static priority level $\left(\mathrm{P}^{\mathrm{L}_{1}}\right)$ of cluster members at the end of each period according to the sleeping or monitoring mode of the members in the current period. The second level of priority of each node is preset in each period for the next period. Exchanging messages for synchronization, getting up-to-date residual energy values $\left(E_{R}\right)$ and maintaining clusters are done as mentioned in section III.A. Recomputing the energy level of nodes at the end of each period is according to the following equations:

$\mathrm{E}_{\mathrm{R}_{\mathrm{i}}}^{\mathrm{k}+1}=\mathrm{E}_{\mathrm{R}_{\mathrm{i}}}^{\mathrm{k}}-\mathrm{E}_{\mathrm{M}}, \forall \mathrm{S}_{\mathrm{i}} \in \mathrm{CSS}$

$\mathrm{E}_{\mathrm{R}_{\mathrm{i}}}^{\mathrm{k}+1}=\mathrm{E}_{\mathrm{R}_{\mathrm{i}}}^{\mathrm{k}}-\mathrm{E}_{\mathrm{S}}, \forall \mathrm{S}_{\mathrm{i}} \notin \mathrm{CSS}$

Fig. 6 shows the selected covering sensors sets awakened in several monitoring periods under the proposed algorithm. As it can be observed, in each period the awakened nodes cover all the clusters of Fig. 4. Table 2 shows the proposed IICC algorithm. The concepts of abbreviations used in the algorithm are as follows:

- $\boldsymbol{C} \boldsymbol{C}$ : Covered Cluster Vector of size M (number of clusters), that shows the covered clusters by the selected multimedia nodes

- PVL1: Level one Priority Vector of size N (number of nodes) that consists of the first level covering priority value of each multimedia node in the network

- PVL2: Level two priority Vector of size N that consists of the second level covering priority value of each multimedia node in the network

- $\boldsymbol{E}_{\text {init }}$ : The initial energy of each node

- CSS: Covering Sensors Set, that consists of the activated sensor nodes covering all clusters

- CCN: The Candidate Covering node for joining to CSS

- $\boldsymbol{E}_{\boldsymbol{M}}$ : The energy consumed by an activated node during the period T

- $\boldsymbol{E}_{S}$ : The energy consumed by a sleeper node during the period $T$

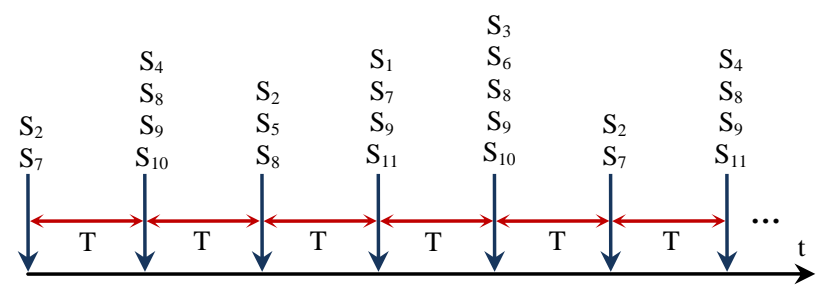

Figure 6. The selected covering sensors sets to monitor the area with Intra and Inter-Cluster cooperation.
Table 2. The second Scheduling Algorithm

Intra and Inter-Cluster Cooperation (IICC)

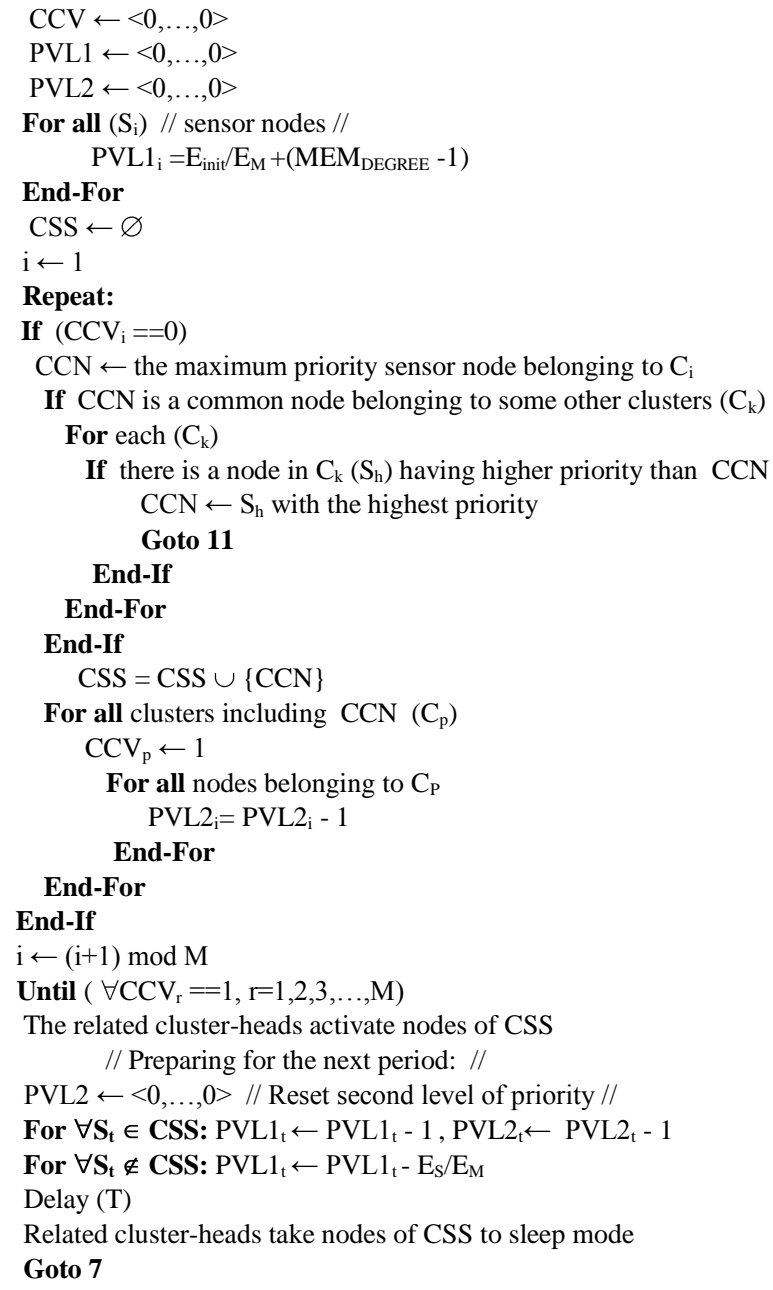

\section{COMPARISONS AND EVALUATIONS}

In order to evaluate the proposed node selection algorithms, we study an un-cooperative periodic monitoring mechanism similar to the first tier of [13] as a baseline to see the benefit of cooperation in power efficiency and compare the proposed algorithms. For generality in the three cases, we assume that monitoring nodes capture an image from their perspective of the area and accomplish the object detection procedure to survey the presence of objects or events. When a node detects an object or event, notifies its cluster-head and thus the sink and also send the image. Without loss of generality, we assume the Cyclops [14] as the camera sensor in the multimedia nodes.

In the case monitoring without coordination between multimedia nodes, all nodes are programmed to independently wake up and monitor the area with a monitoring period of time (T) in a duty-cycled manner [13]. Thus, the nodes consume their stored energy according to their sleep and active modes during each period. Each node sleeps during the time $T-T_{M}$ in each period, where $T_{M}$ is the monitoring time taken for capturing an image and surveying the presence of an 
object/event by the node. We assume that the initial amount of stored energy in all nodes is the same. Thus, as the level of energy in all nodes is decreased with the same rate per monitoring period, the lifetime of the nodes will be the same. Fig. 7 shows the number of alive nodes in a network consisting of 250 multimedia nodes for un-cooperative, intracluster cooperative (ICC) and intra \& inter-cluster cooperative (IICC) methods. We can see that in the un-cooperative mechanism, the network is alive with all of its nodes until their energy will be spent completely. After each T, the level of stored energy in all nodes of the network decrease uniformly and thus the network will die with all of its nodes after the specific number of $\mathrm{T}$ which depends on: (i) the amount of initial stored energy in nodes, (ii) the characteristics of the mote and the embedded multimedia sensor in the nodes, (iii) the period of awakening nodes (T).

Therefore, defining the lifetime of the un-cooperative network as $L_{t}$ as the time to which nodes spent all of their energy, all nodes under the un-cooperative scheme will die at the same time with a difference of $\mathrm{T}$ seconds among them (i.e., $\mathrm{L}_{\mathrm{t}} \pm \mathrm{T}$ ) since the nodes awake asynchronously with the period $\mathrm{T}$. The time axis of the diagram has been normalized by the $\mathrm{L}_{\mathrm{t}}$.

For the cooperative schemes, each down step of the diagram indicates losing some nodes of the network. As the figure shows, the network surveying the environment with IICC algorithm has alive nodes until even $5.5 \mathrm{~L}_{\mathrm{t}}$. Single nodes (clusters having only one node) are the first set of dying nodes (i.e., dying at $\mathrm{L}_{\mathrm{t}}$ ). Then, cluster members of clusters of size two nodes die at $1.83 \cdot \mathrm{L}_{\mathrm{t}}$ and clusters of size three die at $2.52 \cdot \mathrm{L}_{\mathrm{t}}$. As it is observed from the figure, bigger clusters can keep their nodes for a longer time than sparse ones because of having more cooperation capability among the members/clusters. Using only ICC scheme also achieves a good network lifetime increase, $4.5 \mathrm{~L}_{\mathrm{t}}$ with respect the uncooperative scheme. However, the efficiency is not such good as the IICC scheme. As explained in the former subsection, the last scheme benefits from an intelligent schedule from the nodes belonging to more than one cluster.

To reinforce this argument, Fig. 8 shows the number of awakened nodes for monitoring the area during each monitoring period in terms of node density for IICC, only intra-cluster cooperative and the un-cooperative monitoring schemes. The density of the network is varied from a sparse deployment with 50 nodes in the sensing area that is a square of $120 \mathrm{~m} \times 120 \mathrm{~m}$ to a dense case of 300 nodes that covers $95 \%$ of the area. In the un-cooperative monitoring, during each period all sensor nodes will wake up to monitor the area while in the cooperative methods, during each period every cluster select and awaken one representative for monitoring. Thus, in the intra-cluster cooperative scheme the number of active nodes is equal to the number of established clusters in the network while in IICC scheme because of cooperation between clusters, the number of active nodes is less than the number of clusters. In a sparse deployment, overlapping between nodes and also clusters is less than dense ones thus the potential of cooperation in sparse networks is lower than in dense networks. As it can be observed form the figure, the difference between the number of active nodes in the two cooperative schemes and also the difference between both of

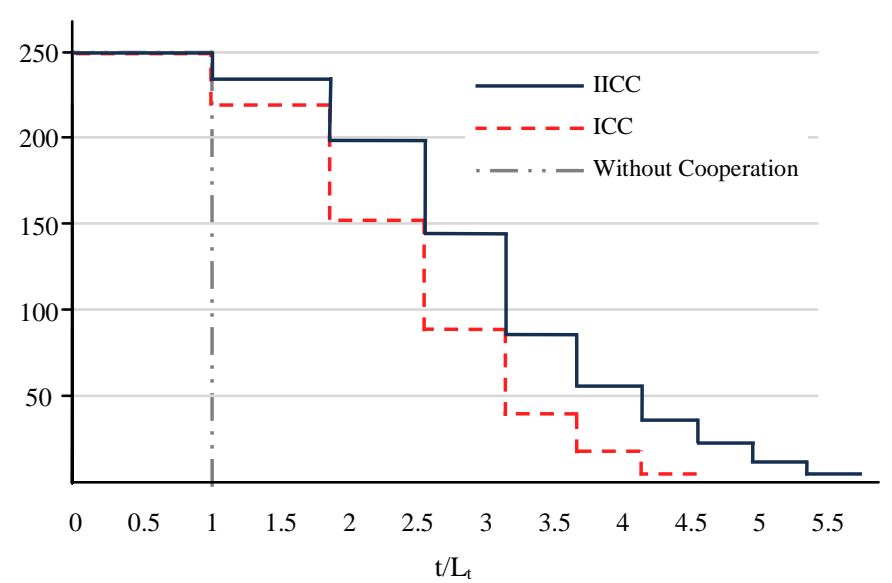

Figure 7. Number of alive nodes in terms of time for both proposed cooperative selection methods and the un-cooperative ordinary scheme

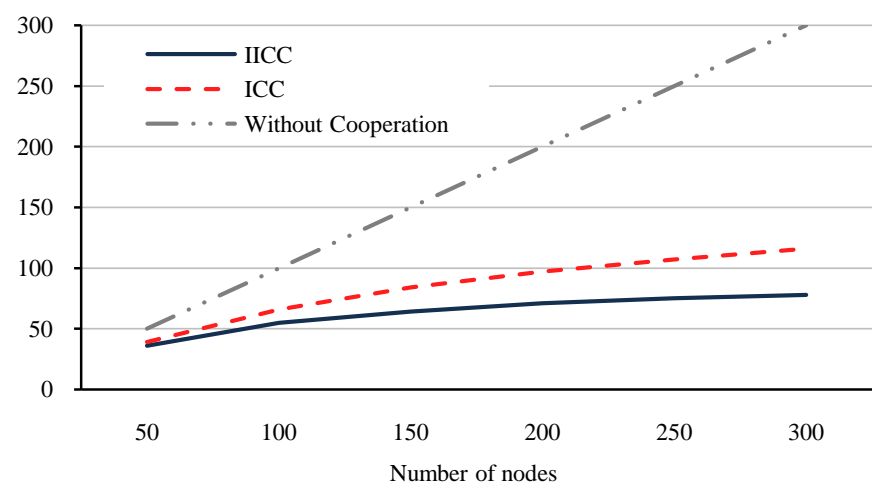

Figure 8. Number of awakened nodes during a monitoring period for both proposed cooperative methods and un-cooperative ordinary scheme

them and the un-cooperative scheme raises with increasing the density of the network. This is the result of increasing cooperation potential.

In the IICC algorithm, overlapping among clusters makes an enhancement in the size of clusters and the collaboration between clusters increases their life-time. As it can be observed in the Fig. 7, at each time, the number of alive nodes in the network with IICC is larger than ICC. Particularly, many of the single nodes that die at the $\mathrm{L}_{\mathrm{t}}$ in ICC method, are joined to other clusters by MCM and cooperate with other cluster members and thus work for a considerable longer time.

However, it is true that the cost of the cooperation schemes come in some extra node communication. In the intra-cluster cooperative algorithm, the cluster-heads needs only to know the stored energy level of their cluster members so each cluster-head keeps the related information in a record and in each monitoring period after the decision about active node, recomputes it based on the decided member states; all sleeper nodes consume $\mathrm{E}_{\mathrm{S}}$ during the current period and the active node consumes $\mathrm{E}_{\mathrm{M}}$. Therefore, it does need to data transfer between the cluster-head and cluster members for keeping the information up to date.

Nevertheless, IICC sometimes obligates cluster-heads (when a cluster-head is deciding about a common node to be an active node) to know the priority level of the members 
belonging to the neighbouring clusters for deciding about active node. For this case, the cluster-head has to connect the cluster-head of the target cluster to obtain all required information of all its members. So, in IICC method, some node information is communicated between neighbouring clusters but it does not have so effect to be addressed as a considerable overflow. For example, in a clustered network consisting of 250 nodes whose average MEM $_{\text {DEGREE }}$ is 1.325 [6], the average number of packets which have to be transmitted between neighbouring clusters is 30 for each period of monitoring. This number of packet transmissions for a multimedia network that deals with transmission of a lot of packets related to multimedia data, is negligible.

\section{CONCLUSIONS}

Wireless sensor networks are battery powered, therefore prolonging the network lifetime through a power aware node organization is highly desirable. An efficient method for energy saving is to schedule the sensor nodes activity such that every sensor alternates between sleep and active state. One solution is to organize the sensor nodes in clusters, such that each cluster monitors its domain. In this paper we proposed priority-based node selection and scheduling mechanisms to apply coordination on cluster members for monitoring their domain cooperatively. Moreover, the overlapping clusters collaboratively employ their common members based on a defined priority to be awakened and monitor the area. When a node is awakened, it captures an image from its perspective and then surveys presence of a new object/event in the image. The coordination within and also between clusters makes a considerable development in energy conservation capability of the nodes and prolongs the network lifetime.

\section{ACKNOWLEDGMENT}

This work has been supported by Spanish Ministry of Science and Technology under grants TSI2007-66869-C02 and 2009 SGR-1167.

\section{REFERENCES}

[1] F. Akyildiz, T. Melodia, and K. R. Chowdhury, "A survey on wireless multimedia sensor networks," Computer Networks (Elsevier) Journal, Vol. 51, pp. 921-960, 2007.

[2] N. Dimokas, D. Katsaros, Y. Manolopoulos, "Cooperative Caching in Wireless Multimedia Sensor Networks," Mobile Network Applications (Springer), vol. 13, pp. 337-356, 2008.

[3] M. Tahir, R. Farrell, "Optimal communication-computation trade off for wireless multimedia sensor network lifetime maximization," In proceedings of the 2009 IEEE conference on Wireless Communications \& Networking Conference (IEEE WCNC), pp. 2514-2519, Budapest, Hungary, April 2009.

[4] S. Soro, W. Heinzelman, "On the coverage problem in video-based wireless sensor networks," In proceedings of the 2nd IEEE International Conference on Broadband Communications and Systems (BroadNets), pp. 932-939, Boston, MA, USA, October 2005.

[5] M. Alaei, J. M. Barcelo-Ordinas, "Node Clustering Based on Overlapping FoVs for Wireless Multimedia Sensor Networks," In proceedings of the IEEE Wireless Communications and Networking Conference (IEEE WCNC), Sydney, Australia, April 2010.

[6] M.Alaei, J.M. Barcelo-Ordinas, "MCM: Multi-Cluster-Membership Approach for FoV-Based Cluster Formation in Wireless Multimedia Sensor Networks," the 6th ACM International Wireless Communication \& Mobile Computing Conference (IWCMC 2010), Caen, France, June 2010.
[7] M. Cardei, M. T. Thai, Y. Li, W. Wu, "Energy-Efficient Target Coverage in Wireless Sensor Networks," In proceedings of the $24^{\text {th }}$ Conference of the IEEE Computer and Communication Societies (IEEE INFOCOM), pp. 1976 - 1984, Miami, Florida, USA, March 2005.

[8] Newell, K. Akkaya, "Self-actuation of Camera Sensors for Redundant Data Elimination in Wireless Multimedia Sensor Networks," In proceedings of the IEEE International Conference on Communications (IEEE ICC), Dresden, Germany, June 2009.

[9] R. Dai, I. F. Akyildiz, "Joint Effect of Multiple Correlated Cameras in Wireless Multimedia Sensor Networks," In proceedings of the IEEE International Conference on Communications (IEEE ICC), Dresden, Germany, June 2009.

[10] X. Wang, J. J. Ma, L. Ding, Bi D. W. Bi, "Robust Forecasting for Energy Efficiency of Wireless Multimedia Sensor Networks," Sensors (MDPI), vol. 7, no. 11, pp. 2779-2807, 2007.

[11] L. Liu, X. Zhang, H. Ma, "Dynamic Node Collaboration for Mobile Target Tracking in Wireless Camera Sensor Networks," In proceedings of the $28^{\text {th }}$ Conference of the IEEE Computer and Communication Societies (IEEE INFOCOM), pp. 1188-1196, Rio de janerio, Brazil, April 2009.

[12] M. P. J. Adriaens, S. Megerian, "Optimal worst-case coverage of directional field-of-view sensor networks," In Proceedings of the $3^{\text {rd }}$ IEEE Communication Society Conference on Sensor, Mesh and Ad Hoc Communications and Networks (IEEE SECON), Reston, VA USA, pp. 336-345, September 2006.

[13] P. Kulkarni, D. Ganesan, P. Shenoy, and Q. Lu, "SensEye: a multi tier camera sensor network," In proceedings of the $13^{\text {th }}$ ACM International Conference on Multimedia (ACM Multimedia), pp. 229 - 238, Hilton, Singapore, November 2005.

[14] M. Rahimi, R. Baer, O. I. Iroezi, J. C. Garcia, J. Warrior, D. Estrin, M. Srivastava, "Cyclops: in situ image sensing and interpretation in wireless sensor networks," In proceedings of the $3^{\text {rd }}$ ACM Conference on Embedded Networked Sensor Systems (ACM SenSys), pp.192 - 204, San Diego, USA, November 2005.

[15] M. Alaei, J. M. Barcelo-Ordinas, "A Cluster-based Scheduling for Object Detection in Wireless Multimedia Sensor Networks," In proceedings of the 5th ACM International Symposium on QoS and Security for Wireless and Mobile Networks (ACM Q2SWinet, collocated with MSWiM), pp. 50-56, Canary Islands, Spain, October 2009.

[16] S. Lindsey, C. S. Raghavendra, "PEGASIS: Power Efficient Gathering in Sensor Information Systems," In proceedings of the IEEE Aerospace Conference, pp. 1125-1130, Big Sky, Montana, March 2002.

[17] S. Ganeriwal, R. Kumar, S. Adlakha, M. Srivastava, "Network-wide time synchronization in sensor networks," Technical Report, Networked and Embedded Systems Lab, Electronic Engineering Department, UCLA, 2003. 